\section{Zink bei ADHS: nur in Kombination wirkungsvoll}

Kann eine Substitution von Zink die ADHS-Symptomatik mindern und führt eine Kombination von Amphetaminsulfat und Zink zu einem positiven additiven Effekt? Diesen Fragen ging ein Team von US-amerikanischen Wissenschaftlern nach.

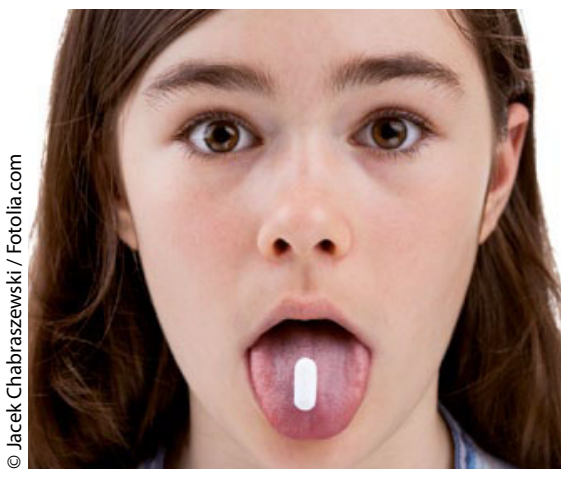

Zink alleine hat bei Kindern mit ADHS keine Wirkung.

nsgesamt 52 Kinder mit Aufmerksamkeitsdefizit-/Hyperaktivitätsstörung (ADHS) im Alter von sechs bis 14 Jahren erhielten in einer Doppelblindstudie einoder zweimal täglich Zink als Glycinat oder Placebo. Nach acht Wochen wurde die Medikation ergänzt durch D-Amphetamin, titriert nach Wirkung, für fünf Wochen. Nach 13 Wochen wurden beide Gruppen miteinander verglichen.

Die Autoren stellten fest, dass Zink alleine im Vergleich zu Placebo keinen Einfluss auf die ADHS-Symptomatik hatte. Wurde Zink hingegen mit Amphetamin kombiniert, konnte die Amphetamindosis um $37 \%$ reduziert werden im Vergleich zu Placebo kombiniert mit Amphetamin. Dieser positive additive Effekt war jedoch nur mit einer zweimaligen Gabe pro Tag von $15 \mathrm{mg}$ Zinkglycinat zu erzielen.

Arnold LE et al. Zinc for attention-deficit/ hyperactivity disorder: placebo-controlled double-blind pilot trial alone and combined with amphetamine. J Child Adolesc Psychopharmacol 2011;21:1-19
Kommentar: Zink ist ein wichtiger Kofaktor im Stoffwechsel von Neurotransmittern, Prostaglandinen und Melatonin. Indirekt beeinflusst es auch den Dopaminstoffwechsel durch Blockade des Dopamintransporters. Bei Zinkmangel wird unter anderem auch hyperaktives Verhalten beschrieben sowohl in Tierversuchen als auch bei Menschen. Ein Einfluss auf die ADHS-Symptomatik ist also vorstellbar, insbesondere bei Vorliegen eines Zinkmangels. Bei den Studienteilnehmern lag jedoch nachweislich kein Zinkmangel vor. Aus der Studie ergeben sich für den klinischen Alltag zwei wichtige Informationen: 1. Eine isolierte Zinksubstitution zeigt keinen Effekt. 2. Eine Reduktion der Amphetamindosis um knapp $40 \%$ durch eine additive Zinktherapie ist vor allem bei unerwünschten Nebenwirkungen der Amphetamintherapie vorteilhaft.

Aus den Studiendaten ist jedoch ebenfalls zu entnehmen, dass es unter der isolierten Zinktherapie bei $15 \%$ der Kinder zu Depressionen, bei $20 \%$ zu abdominellen Beschwerden, bei $30 \%$ zu erhöhter Irritabilität und bei $10 \%$ zu Appetitverlust kam. Also auch hier wieder der alte Grundsatz: ohne Nebenwirkung keine Wirkung. Dr. Kirsten Stollhoff

\section{Thrombopoietin-Mimetika als neue Behandlungsoption bei Kindern mit ITP?}

\begin{abstract}
Wissenschaftler von der Cornell University in New York testeten eine neue Therapieoption bei Kindern mit Immun-Thrombozytopenie: das Thrombopoietin-Mimetikum Romiplostim. Es zeigte sich, dass Romiplostim die Thrombozytenzahl deutlich erhöhen konnte.
\end{abstract}

W ährend die meisten Kinder mit Immun-Thrombozytopenie (ITP) eine spontane Erholung der Thrombozytenzahlen zeigen oder trotz chronischer Verläufe keine Therapie benötigen, bleiben etwa 5-10\% aller pädiatrischen ITPPatienten mit schwerer chronischer Thrombozytopenie behandlungsbedürftig. Die meisten der derzeitigen therapeutischen Optionen wie beispielsweise die regelmäßigen Gaben von Cortison, intravenösen Immunglobulinen oder AntiD-Immunglobulin, des Einsatzes von Immunsuppressiva wie Rituximab oder eine Splenektomie gehen möglicherweise mit beträchtlichen Nebenwirkungen einher.
Bei Erwachsenen mit chronischer ITP zeigten Zweitgeneration-Thrombopoietin-Rezeptor-Agonisten, die die Thrombozytenproduktion stimulieren, ermutigende Ergebnisse. Diese Medikamente sind bisher bei Kindern kaum geprüft und auch nicht zugelassen.

In einer randomisiert und doppelblind durchgeführten, placebokontrollierten Phase-I/II-Multicenter-Studie wurde nun erstmals bei Kindern der Effekt und die Sicherheit des Thrombopoietin-Mimetikums Romiplostim untersucht. Patienten im Alter zwischen zwölf Monaten und 18 Jahren und einer ITP-Dauer von mindestens sechs Monaten wurden in die Studie aufgenommen. 17 Kinder erhielten über insgesamt zwölf Wochen wöchentliche Gaben Romiplostim, fünf Kinder Placebo. Die behandelnden Ärzte versuchten jeweils die Dosis so anzupassen, dass die Thrombozytenwerte zwischen 50.000 und 250.000/ $\mu$ l lagen.

In der Romiplostim-Gruppe erreichten 15 der 17 Kinder Thrombozytenzahlen $\geq 50.000 / \mu$ in zwei aufeinanderfolgenden Wochen, während dies bei keinem der Patienten der Placebo-Gruppe erreicht wurde ( $p=0,0008)$. In der RomiplostimGruppe wurden während der Studienphase von zwölf Wochen Thrombozytenzahlen $>50.000 / \mu \mathrm{l}$ im Mittel über sieben Wochen (Spannbreite 0-11 Wochen) gesehen, während dies unter Placebo überhaupt nicht beobachtet werden konnte $(\mathrm{p}=0,0019)$.

Kein Patient brach die Studie vorzeitig $\mathrm{ab}$, und bei keinem Patienten wurden schwere unerwünschte therapieassoziierte Arzneimittelwirkungen gesehen. Wie bei Erwachsenen waren auch bei Kindern Kopfschmerzen und Nasenbluten die häufigsten Nebenwirkungen. Die Autoren 
folgern aus den Ergebnissen dieser Studie, dass Romiplostim bei der überwiegenden Anzahl der Kinder mit ITP die Thrombozytenzahl deutlich erhöhen kann und gut vertragen wird.

Bussel JB et al. A randomized, double-blind study of romiplostim to determine its safety and efficacy in children with immune thrombocytopenia. Blood 2011; 118: 28-36
Kommentar: Die Ergebnisse der Studie zeigen eindrucksvoll, dass ein ThrombopoietinMimetikum wie Romiplostim durchaus eine Therapieoption bei Kindern mit chronischer ITP sein kann. Allerdings ist die Behandlungssowie Nachbeobachtungszeit der Studie zu kurz, um später auftretende unerwünschte Nebenwirkungen des Medikaments zu erfassen. Weiterhin muss die Indikation für Thrombopoietin-Mimetika kritisch diskutiert werden. Da die meisten Kinder mit ITP im Verlauf ihrer Erkrankung keine schwere Blutung erleiden, kann die Wirksamkeit eines Medikamentes nicht allein durch die Anhebung der Thrombozytenzahl bewiesen werden, sondern es muss die Effektivität des Medikamentes durch eine Verringerung der Inzidenz schwerer Blutungen bzw. der Letalität nachgewiesen werden. Dies kann jedoch nur wenn überhaupt - in großen internationalen Studien gezeigt werden.

Prof. Dr. Thomas Lehrnbecher

\section{Bocaviren als häufige Ursache einer Pneumonie bei Kindern}

Das humane Bocavirus (HBoV) ist ein neu identifiziertes Parvovirus, das häufig bei Kindern mit akuten Atemwegs- und Magen-Darm-Infektionen zu finden ist. Seine Bedeutung für die kindliche Pneumonie ist bisher unbekannt.

Ü ber einen Zeitraum von 15 Monaten wurden prospektiv 124 Kinder im Alter von einem Monat bis 15 Jahren, die bei Verdacht auf eine Pneumonie in Norditalien stationär aufgenommen worden waren, mithilfe serologischer Antikörpertests untersucht. Dabei wurden 16 verschiedene Mikroorganismen erfasst. 101 Kinder hatten eine radiologisch bestätigte Pneumonie.

Nach RSV-Infektionen, die bei $17 \%$ der Kinder auftraten, war eine HBoV-Infektion die zweithäufigste Ursache (12\%) für die Pneumonie. Sieben Kinder waren ausschließlich mit HBoV infiziert, während fünf Kinder gemischte Infektionen mit anderen Viren oder mit Bakterien aufwiesen. Elfmal wurde die Diagnose durch ein erhöhtes IgM, sechsmal durch einen Anstieg von IgG gestellt. Die Seropositivität für $\mathrm{HBoV}$ stieg altersabhängig und hatte mit Ende des Vorschulalters fast $100 \%$ erreicht. $\mathrm{HBoV}$ sind damit eine häufige Ursache der Pneumonie bei Säuglingen und Kleinkindern.

Don $\mathrm{M}$ et al. Serologically verified human Bocavirus pneumonia in children. Pediatr Pulmonol 2010; 45: 120-6

\section{Was der Blick in den Hals bei V.a. Streptokokken verrät}

Eine Streptokokken-Pharyngitis bei Kindern taugt nicht zur Blickdiagnose: Es gibt keine Symptome oder Krankheitszeichen, die - für sich allein oder in Kombination - die Infektion durch Streptokokken definitiv sichern oder ausschließen können. Trotzdem gibt es einige Befunde, die zumindest in Richtung Streptokokken-Infektion weisen. In einer systematischen Übersichtsarbeit wurden alle Veröffentlichungen aus den Jahren 1953 bis 2010 ausgewertet, die sich mit der Aussagekraft von Symptomen der StreptokokkenPharyngitis beschäftigten.

Es kristallisierten sich fünf Symptome heraus, bei denen immerhin eine über 50\%ige Wahrscheinlichkeit für eine Streptokokken-Pharyngitis bestand: 1. scarlatiniformes Exanthem, 2. palatinale Petechien, 3. pharyngeales Exsudat, 4. Erbrechen und 5. druckempfindliche Halslymphknoten. Einen deutlichen Hinweis auf ein geringes Streptokokken-Risiko liefert einer Studie zufolge die Kombination aus mindestens mittelschwerer Erkältung, höchstens leicht geschwollenen Halslymphknoten und Tonsillen und dem Fehlen eines scarlatiniformen Exanthems.

Dr. Beate Schumacher

Kommentar: HBoV sind nach RSV und Rhinoviren die dritthäufigsten Viren, die bei kleinen Kindern mit einer obstruktiven Bronchitis gefunden werden. Bei etwa 70-90\% der Kinder mit akutem Giemen finden sich im Rahmen einer virusinduzierten Atemwegsobstruktion serologische Hinweise auf HBoV. Bei etwa 10-20\% können in diesen Situationen HBoV aus den Atemwegssekreten identifiziert werden. Vorläufige seroepidemiologische Daten deuteten auf die Bedeutung von $\mathrm{HBoV}$ hin, da IgG-Antikörper bei über $90 \%$ der unter Sechsjährigen nachgewiesen werden.

Außerdem wird deutlich, dass entscheidend auf die Methodik und Definition der Infektion geachtet werden muss. Untersuchungen die nur auf dem Nukleinsäurenachweis (PCR) aus Sekreten beruhen und bei denen oft simultan weitere Viren gefunden werden, können die genaue pathogenetische Rolle von HBoV nicht einordnen.

Obgleich RSV-Infekte insbesondere zwischen November und Januar häufig vorkamen, hatte nur ein Kind eine Mischinfektion mit HBoV und RSV. Dies spricht für die unabhängige pathogenetische Bedeutung von HBoV für Pneumonien.

Dies ist die erste Studie, die klare Belege für die Rolle von HBoV bei Pneumonien liefert. Eine vorherige PCR-Untersuchung hatte das Virus bei 3,9\% der kindlichen Pneumonien identifiziert. Alle HBoV-positiven Pneumonien traten bei Kindern unter vier Jahren auf. Eine fast komplette Serokonversion ist bis zum Alter von sechs Jahren erreicht. Inwieweit die Antikörper protektiv wirken oder erneute Infektionen auftreten können, ist noch offen.

Prof. Dr. Matthias Griese

Shaikh N et al. J Pediatr 2011 Oct 31. [Epub ahead of print] 